\title{
Use of an Accurate DNS Particulate Flow Method to Supply and Validate Boundary Conditions for the MFIX Code
}

\author{
FINAL REPORT \\ June 2009 ----- May 2012 \\ Prepared for \\ U.S. Department of Energy \\ National Energy Technology Laboratory \\ 3610 Collins Ferry Road \\ P.O. Box 880 \\ Morgantown, WV 26507-0880 \\ Grant Number: DE-NT0008064 \\ Prepared by \\ Zhi-Gang Feng \\ University of Texas at San Antonio \\ Mechanical Engineering \\ Tel: 210-458-5737 \\ Fax: $210-458-6504$ \\ zhigang.feng@utsa.edu \\ September 24, 2012
}




\section{DISCLAIMER}

This report was prepared as an account of work sponsored by an agency of the United States Government. Neither the United States nor any agency thereof, nor any of their employees, makes any warranty, express or implied, or assumes any legal liability or responsibility for the accuracy, completeness, or usefulness of any information, apparatus, product, or process disclosed, or represents that its use would not infringe privately owned rights. Reference herein to any specific commercial product, process, or service by trade name, trademark, manufacturer, or otherwise does not necessarily constitute or imply its endorsement, recommendation, or favoring by the United States Government or any agency thereof. The views and opinions of authors expressed herein do not necessarily state or reflect those of the United States Government or any agency thereof. 


\section{ABSTRACT}

The simulation of particulate flows for industrial applications often requires the use of two-fluid models, where the solid particles are considered as a separate continuous phase. One of the underlining uncertainties in the use of the two-fluid models in multiphase computations comes from the boundary condition of the solid phase. Typically, the gas or liquid fluid boundary condition at a solid wall is the so called no-slip condition, which has been widely accepted to be valid for single-phase fluid dynamics provided that the Knudsen number is low. However, the boundary condition for the solid phase is not well understood. The no-slip condition at a solid boundary is not a valid assumption for the solid phase. Instead, several researchers advocate a slip condition as a more appropriate boundary condition. However, the question on the selection of an exact slip length or a slip velocity coefficient is still unanswered. Experimental or numerical simulation data are needed in order to determinate the slip boundary condition that is applicable to a two-fluid model.

The goal of this project is to improve the performance and accuracy of the boundary conditions used in two-fluid models such as the MFIX code, which is frequently used in multiphase flow simulations. The specific objectives of the project are to use first principles embedded in a validated Direct Numerical Simulation particulate flow numerical program, which uses the Immersed Boundary method (DNS-IB) and the Direct Forcing scheme in order to establish, modify and validate needed energy and momentum boundary conditions for the MFIX code. To achieve these objectives, we have developed a highly efficient DNS code and conducted numerical simulations to investigate the particle-wall and particle-particle interactions in particulate flows. Most of our research findings have been reported in major conferences and archived journals, which are listed in Section 7 of this report. In this report, we will present a brief description of these results. 


\section{TABLE OF CONTENTS}

\section{Section}

1. Executive Summary 5

2. Introduction 6

3. Numerical Simulation Method 7

3.1. Direct Numerical Simulation (DNS) 7

3.2. Particle Collision Model 10

4. Results and Discussions 11

$\begin{array}{ll}\text { 5. Conclusions } & 19\end{array}$

$\begin{array}{ll}\text { 6. References } & 21\end{array}$

7. List of Publications 23

$\begin{array}{ll}\text { 7.1 Archival Journal Publications } & 23\end{array}$

7.2 Conference Publications and Presentations 24

8. List of Personals Participated in the Project 25 


\section{EXECUTIVE SUMMARY}

The simulation of particulate flows for industrial applications often requires the use of two-fluid models, where the solid particles are considered as a separate continuous phase. One of the underlining uncertainties in the use of the two-fluid models in multiphase computations comes from the boundary condition of the solid phase. Typically, the gas or liquid fluid boundary condition at a solid wall is the so called no-slip condition, which has been widely accepted to be valid for single-phase fluid dynamics provided that the Knudsen number is low. However, the boundary condition for the solid phase is not well understood. The no-slip condition at a solid boundary is not a valid assumption for the solid phase. Instead, several researchers advocate a slip condition as a more appropriate boundary condition. However, the question on the selection of an exact slip length or a slip velocity coefficient is still unanswered. Experimental or numerical simulation data are needed in order to determinate the slip boundary condition that is applicable to a two-fluid model.

We have completed the modification of the DNS code that tracks the solid particles in the simulated fluidized bed reactor and has obtained data on the isothermal behavior of particles close to the walls. Particle-particle and particle-wall collisions have also been investigated and it is found that the collision input parameters can have significant effects to the dynamics of particle motions. We have developed a method for selecting appropriate collision model parameters. We have derived boundary velocity conditions for the particles, which imply that there is significant particle "slip" on the walls. The slip is in the range 0.3 to 0.5 of the terminal velocity of the particle. It appears from the results that the wall slip depends on the size of the particles, and details of the wall collision process do not play an important role. We have also investigated the motion of a number of particles near a vertical solid wall, while the particles are in fluidization by a uniform flow. By applying the DNS, the positions and velocities of particles, initially randomly distributed in a $0.20 \mathrm{~m} \times 0.80 \mathrm{~m}$ bed, are being tracked and analyzed at each time step. It is found that the motion of particles is highly unsteady. However, the time- and vertical-space averaged values of the particle velocities converge, yielding velocity profiles that can be used to deduce the particle slip length close to a solid wall. The simulation results show a significant amount downward particle slip close to a vertical wall, with the slip velocity increasing as the fluidization velocity increases. However, a negative slip length of approximately 1.2 particle diameters appears to exist. This slip length was found to be insensitive to the values of the fluidization velocity. Theoretical studies have also been conducted to solve some of the fundamental problems related to particulate flows such as the drag-law on single particles under different flow conditions.

We report some of the specific results we have obtained for this project.

Final Report for Grant No. DE-NT0008064 


\section{INTRODUCTION}

Several flow problems in industry and nature involve the flow of a viscous fluid (gas or liquid) with suspended solids. To better understand the dynamics of particulate flows and to aid in the design processes and systems involving particulate flows applications such as fluidized bed reactors, computational simulations provide an efficient, accurate, and inexpensive tool. In the past, researchers have primarily applied two models for studying particulate flows: the classical Eulerian-Eulerian continuum model or two-fluid model (Ishii, 1975; Drew and Lahey, 2001) and the Eulerian-Lagrangian model (Crowe, 1982). The Two-Fluid Model (TFM) treats the dispersed solid phase as a separate continuum fluid with its own set of governing equations for the momentum equations; both phases have their own physical properties. The traditional Eulerian-Lagrangian model is often called the Distinct Element Method (DEM) or the UnResolved Discrete Particle Method (URDPM) (van der Hoef et al., 2008); it treats the fluid as a continuum phase and the solid phase as a collection of discrete particles that obeys Newton's $2^{\text {nd }}$ law. To determine the velocity and position of a particle, one needs to have the correct value of all the forces acting on the particle, which requires empirical equations for the drag and lift coefficients. Another numerical approach for studying particulate flows is the Direct Numerical Simulation (DNS) method, also termed as the Resolved Discrete Particle Method (RDPM). This approach resembles the DEM; however, the DNS determines the drag forces on the particles directly by modeling the particles in the flow and solving the fluid governing equations with the proper boundary conditions on the fluid-particle interfaces. Several numerical schemes that can be classified as DNS have been developed in the past decade, including mesh adaption based methods (Hu et al., 1992; Feng et al., 1994); more efficient Immersed Boundary (IB) based methods (Peskin, 1977; Feng and Michaelides, 2005, 2005; Uhlmann, 2005) and fictitious domain method (Glowinski et al., 1999). The DNS has been recently extended to include particle-fluid heat interactions by obtaining the fluid temperature fields surrounding the particles and computing from the energy equation the heat transfer rate directly (Yu et al, 2006; Feng and Michaelides, 2008, 2009a). By taking into account the energy interactions, the DNS method can successfully model the natural convection that occurs around the particles during particulate heat transfer and combustion processes.

As with the solution of all differential equations, the boundary conditions at the wall play a very important role in the accuracy of the results of two-fluid models. Essentially, the boundary conditions define the solution of the particulate flow system. However, there have been very few studies that address quantitatively the problem of the definition of the boundary conditions at the wall for the particulate phase in two-fluid models. Clearly, because of the kinematic non penetration condition, the normal component of the particulate velocity at the wall must be zero, but there is no a priori reason for the tangential velocity component to vanish. Actually, most of 
the evidence, which includes experimental work and numerical simulations, shows that the tangential no-slip condition is not the right boundary condition for the particulate phase close to solid walls (Johnson and Jackson, 1987; Sommerfeld and Huber, 1999; Michaelides, 2006; Lauga, et al., 2007). This phenomenon is also corroborated by the molecular dynamics simulations on the interactions of spheres/molecules with walls in rarefied flows. While the noslip boundary condition at the walls has been the standard boundary conditions for fluid flows, it has been known since the 1920's that there is slip at the walls in rarefied flows (Lauga et al, 2007). It is apparent that more information is needed for the behavior of particles near the boundary walls, which will enable us to extract meaningful and accurate boundary conditions for the particulate phase. This information may come from experimental data and DNS studies. The detailed results from the DNS have been proved to be useful in extracting constitutive equations and closure laws that can be employed in DEM or TFM. Pan et al. (2002) conducted a DNS of the fluidization of 1204 spherical particles. They were able to successfully simulate the experiment and reproduce the celebrated correlations of Richardson and Zaki (1954), which account for the concentration of particles in the sedimentation process.

The present study pertains to the behavior of solid particles close to a vertical wall from which the normal and tangential velocities of individual particles may be calculated. We have developed a DNS method to simulate the motions of particles in a Fluidized Bed Reactor (FBR) and applied this method to groups of particles that circulate in the FBR. From the flow information obtained, we studied the velocities and the overall motion of the particles in the FBR and extracted the time-averaged velocity profile of the particles. The results of the calculations enable us to determine the tangential component of the velocity of the solid phase and its gradient close to the wall and to deduce the slip wall boundary condition that may be used in two-fluid models.

\section{NUMERICAL SIMULATION METHOD}

\subsection{Direct Numerical Simulation (DNS)}

The DNS method that is applied in this study makes use of the IB method takes into account the natural convection around the particles and has been described before by Feng and Michaelides $(2008,2009)$. However, because we wish to study the boundary conditions without the heat transfer complications, in this study we only use the isothermal part of the DNS method. The IB makes use of two geometric domains: the domain of the entire fluid, $\Omega$, and the domain occupied by the solid particles, $\Sigma\left(\mathrm{S}_{\mathrm{i}}\right)$.

The governing equations for the motion of the fluid and of the particles are as follows:

\subsubsection{For the velocity field in the entire domain, $\Omega$ :}

Final Report for Grant No. DE-NT0008064 
$\frac{\partial \vec{u}}{\partial t}+\vec{u} \cdot \vec{\nabla} \overrightarrow{\mathrm{u}}=-\vec{\nabla} \mathrm{p}+\frac{1}{\mathrm{Re}} \nabla^{2} \overrightarrow{\mathrm{u}}+\overrightarrow{\mathrm{f}}, \overrightarrow{\mathrm{x}} \in \Omega$,

where $\mathrm{f}$ is the fictitious force exerted by the particles on the fluid domain and the Reynolds number, Re is based on the characteristic velocity and length of the problem:

$R e=\frac{\rho_{f} u_{r e f} l_{r e f}}{\mu_{f}}$

3.1.2 For the force density field, in the domain occupied by each particle $S_{i}$ :

$\overrightarrow{\mathrm{f}}=\frac{\partial \vec{u}}{\partial t}+\vec{u} \cdot \vec{\nabla} \overrightarrow{\mathrm{u}}+\vec{\nabla} \mathrm{p}-\frac{1}{\mathrm{Re}} \nabla^{2} \overrightarrow{\mathrm{u}}, \overrightarrow{\mathrm{x}} \in \sum \mathrm{S}_{\mathrm{i}}$

3.1.3 Continuity equation for the entire flow domain $\Omega$ :

$\vec{\nabla} \cdot \overrightarrow{\mathrm{u}}=0, \overrightarrow{\mathrm{x}} \in \Omega$

3.1.4 For the velocity field inside the region occupied by the solid particles, $\Sigma\left(S_{i}\right)$ :

$\vec{u}=\vec{U}_{p}+\vec{\omega}_{p} \times\left(\vec{x}-\vec{x}_{p}\right)$,

where $\vec{x}_{p}$ is the position vector of the center of mass of the particle. Eqn. (5) ensures that the particles undergo a solid body motion. Based on the results for the entire flow domain, the linear and angular velocities of each particle in the flow field are computed at every step of the simulation according to the following expressions:

\subsubsection{For the translational motion of the particles:}

The particle velocity is obtained from the force balance around the fluid at the interface with the particle. The force balance results in the following expression:

$\rho_{p} V_{p} \frac{d \vec{U}_{p}}{d t}=\rho_{f} \oint_{\partial s} \tilde{\sigma} \cdot d \vec{s}+\int_{s}\left(\rho_{p}-\rho_{f}\right) \vec{g} d v$,

where $\rho_{p}, \rho_{f}, V_{p}, \vec{U}_{p}, \tilde{\sigma}$ are particle and fluid density, particle volume, linear velocity and fluid stress tensor respectively.

The surface integral of Equation (6) may be evaluated with the application of the Cauchy theorem and becomes:

$\rho_{f} \oint_{\partial s} \tilde{\sigma} \cdot d \vec{s}=\rho_{f} \int_{s} \vec{f} d v+\rho_{f} \frac{d}{d t} \int_{s} \vec{u} d v$

The time derivative of the volume integral in the last expression may be simplified as follows:

$\frac{d}{d t} \int_{s} \rho_{f} \vec{u} d v=\rho_{f} V_{p} \frac{d \vec{U}_{p}}{d t}$

Substituting Equations (7) and (8) into Equation (6) the following expression for the linear motion of the particle may be derived:

$\left(\rho_{p}-\rho_{f}\right) V_{p} \frac{d \vec{U}_{p}}{d t}=\rho_{f} \int_{s} \vec{f} d v+\left(\rho_{p}-\rho_{f}\right) V_{p} g \vec{e}_{y}$,

where $g$ is the gravitational acceleration constant and $\vec{e}_{y}$ is the unit vector in the direction of the

Final Report for Grant No. DE-NT0008064 
gravity force.

3.1.6 For the rotational motion of the particles:

The angular velocity of the particles is given by the balance of the moments around the particles as follows:

$\frac{1}{2} \pi r^{4} \rho_{p} \frac{d \vec{\omega}_{p}}{d t}=\rho_{f} \oint_{\partial s}\left(\vec{x}-\vec{x}_{p}\right) \times(\tilde{\sigma} \bullet d \vec{s})$,

where $\vec{\omega}_{p}$ and $r$ are the angular velocity and the radius of the particle respectively.

Applying the Cauchy theorem to the surface integral we derive the following expression:

$\rho_{f} \oint_{\partial s}\left(\vec{x}-\vec{x}_{p}\right) \times(\tilde{\sigma} \bullet d \vec{s})=-\rho_{f} \int_{s}\left(\vec{x}-\vec{x}_{p}\right) \times \vec{f} d v+\rho_{f} \frac{d}{d t} \int_{s}\left(\vec{x}-\vec{x}_{p}\right) \times \vec{u} d v$

Taking the time derivative of the volume integral results in the following expression:

$\rho_{f} \frac{d}{d t} \int_{S}\left(\vec{x}-\vec{x}_{p}\right) \times \vec{u} d v=\frac{1}{2} \pi r^{4} \rho_{f} \frac{d \vec{\omega}_{p}}{d t}$

Substituting Equations (11) and (12) into Equation (10) yields the following expression for the angular velocity of the particles:

$\frac{1}{2} \pi r^{4}\left(\rho_{p}-\rho_{f}\right) \frac{d \vec{\omega}_{p}}{d t}=-\rho_{f} \int_{s}\left(\vec{x}-\vec{x}_{p}\right) \times \vec{f} d v$

For the two-dimensional case examined here, the volume of the particles is:

$V_{p}=\pi r^{2}$

The initial conditions of the above set of equations are:

$\vec{U}_{p}(t=0)=\vec{U}_{p 0} ; \vec{\omega}_{p}(t=0)=\omega_{p 0} ;$ and $\vec{u}(t=0)=\vec{u}_{0}$

The subscript 0 represents the initial value $(\mathrm{t}=0)$.

The heat transfer mechanisms are incorporated in a method that is similar to the Immersed Boundary Method (IBM) for the particles: Similar to the momentum interaction for the IBM, the temperature field of the entire region is governed by the following modified energy equation:

$\rho_{f} c_{f} \frac{\partial T}{\partial t}+\rho_{f} c_{f} \vec{u} \bullet \nabla T=k_{f} \nabla^{2} T+q_{p}+\lambda, \quad \vec{x} \in \Omega$,

where, $c_{\mathrm{f}}$ and $k_{\mathrm{f}}$ are the specific heat and thermal conductivity of fluid and $q_{p}$ represents any energy sources inside the particle. The additional energy density term $\lambda$ is added to enforce the temperature field in the region occupied by the particles. As in the case of the force balance equation, this term is zero in the field occupied by the fluid. In the solid region this term is computed using the following equation:

Final Report for Grant No. DE-NT0008064 


$$
\lambda=\rho_{f} c_{f} \frac{\partial T}{\partial t}+\rho_{f} c_{f} \vec{u} \bullet \nabla T-k_{f} \nabla^{2} T-q_{p}, \quad \vec{x} \in \sum S_{i},
$$

where $\mathrm{T}$ is the temperature of each particle. The numerical technique for enforcing the condition of $T=T_{p}(t)$ in the solid region will be discussed in a subsequent section.

The transient temperature of the particles, $T_{p}(t)$, is determined by solving the following differential equation, which is obtained from the energy balance for the particle:

$\rho_{p} V_{p} c_{p} \frac{d T_{p}}{d t}=\oint_{\partial S} k_{f} \vec{\nabla} T_{f} \bullet \vec{n} d s+\int_{S} q_{p} d v$

where $\vec{n}$ is the outward normal vector.

The DNS code was modified to include the heat transfer effects from the particles and the ensuing natural convection. The latter is described by the Grashoff number:

$$
G r=\frac{g \beta L_{r e f}}{U_{r e f}^{2}}\left(T_{p 0}-T_{f 0}\right)\left(\frac{\rho_{f} U_{r e f} L_{r e f}}{\mu_{f}}\right)
$$

\section{3,2 Particle collision model and model parameters}

Particle-particle and particle-wall collisions are inevitable in a particulate flow, especially when the flow is dense and the particles are moving at high velocities. The correct handling of these collisions in any discrete particle method (DEM or DNS) is critical for modeling these types of flows. In general, a collision model is necessary in order to account for the force during collision processes. Without such a mechanism, it is likely that the particles will penetrate significantly into each other's computational boundary, thus, rendering the results meaningless. Most of the collision schemes fall into three categories based on the gap between two particles: repulsive force, hard-sphere, and soft-sphere schemes. Repulsive scheme normally doesn't allow solid-solid overlapping. It uses a pre-defined safe zone. When the gap between two particles is below the safe zone, a repulsive force arises and is added to the particles (Glowinski et al., 1999). The hard-sphere model was first proposed by Alder and Wainwright to study phase transitions in molecular systems (Alder and Wainwright, 1957, 1959). Collision occurs when the gap between two particles is zero; as a result, the collision force and torque are computed and added to the particles. In hard-sphere model, collisions are processed one at a time and a list of future collision candidates has to be maintained for identifying the next collision. This model may lead to very small simulation time step and become inefficient. The soft-sphere model was originally introduced by Cundall and Strack in their work on DEM (1979). In such a model, the particles in contact are allowed to have a small overlapping. The collision forces are computed based on the overlapping displacement. Different force schemes can be used in the model. A 
review of various choices of force schemes can be found in literature (Schafer et al., 1996). Among these schemes, the linear spring/dash-pot model is the most computationally efficient and popular one (Tsuji, 1993; Kuo et al., 2002; Renzo, and Maio, 2004).

The particle-particle collision model that was used in the simulations is the so-called "soft-sphere collision scheme," which involves a linear spring-dashpot model (Feng et al., 2010). The particle-wall collisions are similar to the interparticle collisions: for this purpose, the wall is modelled as a very large particle of infinite radius. The contact forces during such collision processes are composed of two parts: the normal and the tangential components.

In the soft sphere model, the particles are allowed to slightly overlap and the spring model responds instantly by applying a force linearly proportional to the overlap. The force acts quickly and separates the overlapping particles. In the linear spring-dashpot model the normal and tangential components of the contact force may be written as follows:

$f_{i j}^{n}=-k_{n} \delta_{i j}^{n}-\eta_{n} v_{i j}^{n}$

and

$f_{i j}^{t}=-k_{t} \delta_{i j}^{t}-\eta_{t} v_{i j}^{t}$,

where $k_{n}$ and $\eta_{n}$ are the normal spring stiffness and the normal damping coefficient respectively; $\delta_{i j}^{n}$ and $v_{i j}^{n}$ are the normal overlapping displacement and the relative normal velocity component of particle $j$ with respect to particle $i$; similarly, $k_{t}, \eta_{t}, \delta_{i j}^{t}$ and $v_{i j}^{t}$ are the corresponding tangential parameters for the particles $j$ and $i$.

From elementary mechanics, the relative tangential velocity is computed as follows:

$\vec{v}_{i j}^{t}=\vec{v}_{i j}-\left(\vec{v}_{i j} \cdot \hat{n}_{i j}\right) \hat{n}_{i j}+\left[\vec{w}_{i} \times r_{i} \vec{n}_{i j}-\vec{w}_{j} \times r_{i}\left(-\vec{n}_{i j}\right)\right]$

where $\vec{w}_{i}$ is the angular velocity of the particle $i$. The soft sphere collision model may also involve friction during the contact of the particles. The friction is modelled by the coefficients of static and dynamic friction $\mu_{s}$ and $\mu_{k}$ respectively. From this, the tangential contact force may be evaluated from the following expression:

$f_{i j}^{t}=\left\{\begin{array}{lll}-k \delta_{i j}^{t}-\eta v_{i j}^{t}, & \text { if } & \left|f_{i j}^{t}\right| \leq \mu_{s}\left|f_{i j}^{n}\right| \\ \mu_{k}\left|f_{i j}^{t}\right| \frac{\delta_{i j}^{t}}{\left|\delta_{i j}^{t}\right|}, & \text { if } & \left|f_{i j}^{t}\right|>\mu_{s}\left|f_{i j}^{n}\right|\end{array}\right.$

Because of lack of definitive experimental data, several studies use ad hoc assumptions for the determination of these constants. For example, Balevičius et al., (2008) used a single value for the static and dynamic coefficients of friction, $\mu_{\mathrm{s}}=\mu_{\mathrm{k}}=\mu$. In this study we have used the same assumption, with the friction coefficient $\mu=0.3$. Also, the particle and wall materials are assumed to be isotropic and for this reason, the normal and tangential parameters of the stiffness and the damping coefficient are chosen to be equal, and denoted by $\mathrm{k}$ and $\eta$ respectively. Therefore, we have: $k=k_{n}=k_{t}$ and $\eta=\eta_{n}=\eta_{t}$. 
As may be seen in the soft-sphere collision model, there are three parameters that need to be specified:

- the spring stiffness $\mathrm{k}$;

- the damping coefficient $\eta$; and

- the friction coefficient $\mu$.

We have studied the selection of these parameters in the case of a central particle-wall collision in a viscous fluid and concluded that the selection of these collision parameters can be determined by matching the simulation data to the experimentally measured rebounding velocity and collision or contact time of a particle-wall collision.

\section{4. $\quad$ RESULTS AND DISCUSSIONS}

We have developed a multi-level approach that allows us to investigate the slip velocity and boundary conditions at the solid wall of a fluidized bed using the detailed DNS results. We have studied the particle slip velocity at walls in the case of jet flow induced particle fluidizations; we have investigated the relationship between the fluidization velocity and the solid fraction.

According to the Richardson-Zaki (1954) correlation, the fluidization velocity $V(\phi)$ is related to the solid fraction as follows:

$V(\phi)=V(0) f(\phi)$

where $V(0)$ is the fluidization velocity for a single particle, and $f(\phi)$ is a hindered settling function. It is expected that all the particles will be carried out of the bed at the terminal velocity $V(0)$. The hindered function can be correlated by a power law of the fluid fraction,

$f(\phi)=(1-\phi)^{n}=\varepsilon^{n}$

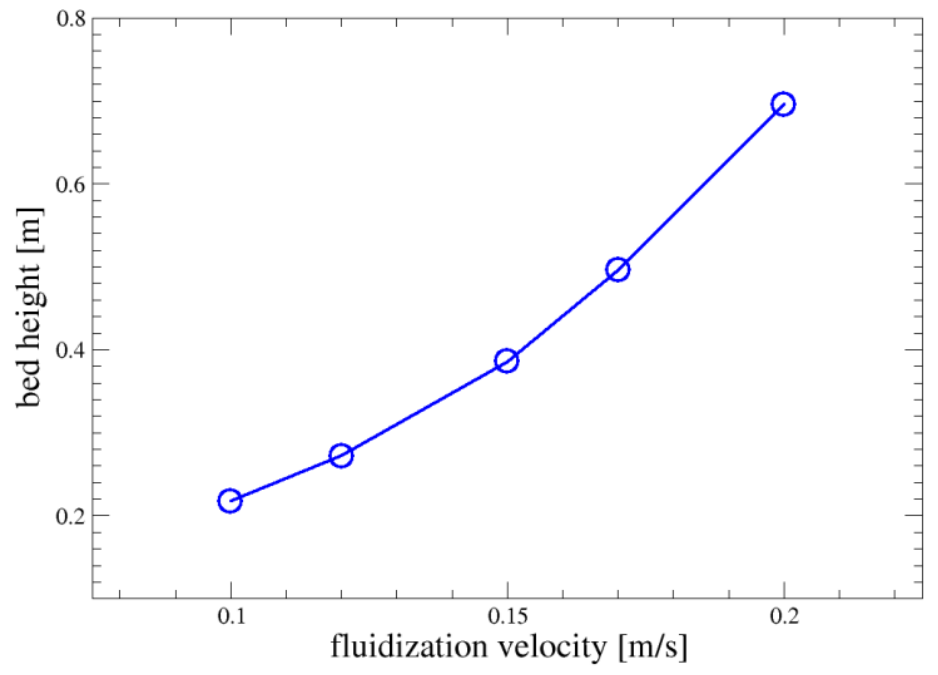

Final Report for Grant No. DE-NT0008064 
Figure 1: Level of fluidized bed at different fluidization velocities

In Figure 1, we plot the data of fluidization velocity and the fluid fraction for the five cases considered. We determined the line that best correlated these data to be,

$V(\phi)=0.27(1-\phi)^{4.24}$

We found that the exponent coefficient in the power-law for a circular particle is $n=4.24$, which is also very significant.

We investigated the slip coefficient of particulate flows in different setups. We believe that the particle slip velocity on a wall depends on the type of flows. Thus, we studied the fluidization of particles by a jet flow. The bed is chosen to be $20 \mathrm{~cm}$ wide and $100 \mathrm{~cm}$ high; the orifice width of the jet is of $1 \mathrm{~cm}$ and jet velocity is of $150 \mathrm{~cm} / \mathrm{s}$. Initially, 1,000 particles are randomly distributed in the half of the lower bed. Using the techniques outlined previously, we computed the time- and space- averaged particles velocity profiles at time $t=0.5 \mathrm{~s}, 5 \mathrm{~s}$, and $10 \mathrm{~s}$, respectively; the results are shown in Figure 2. It is seen that the particles near the wall initially are not moving even though particles further away from the walls are falling because of the gravity. However, after more than 5 seconds, the averaged particles velocity profile becomes relatively steady. Our results show that there is a significant downward slip velocity at both the right and left walls, which is about $0.16 \mathrm{~m} / \mathrm{s}$ or close to an half of the maximum particle velocity found near the center of the bed. 


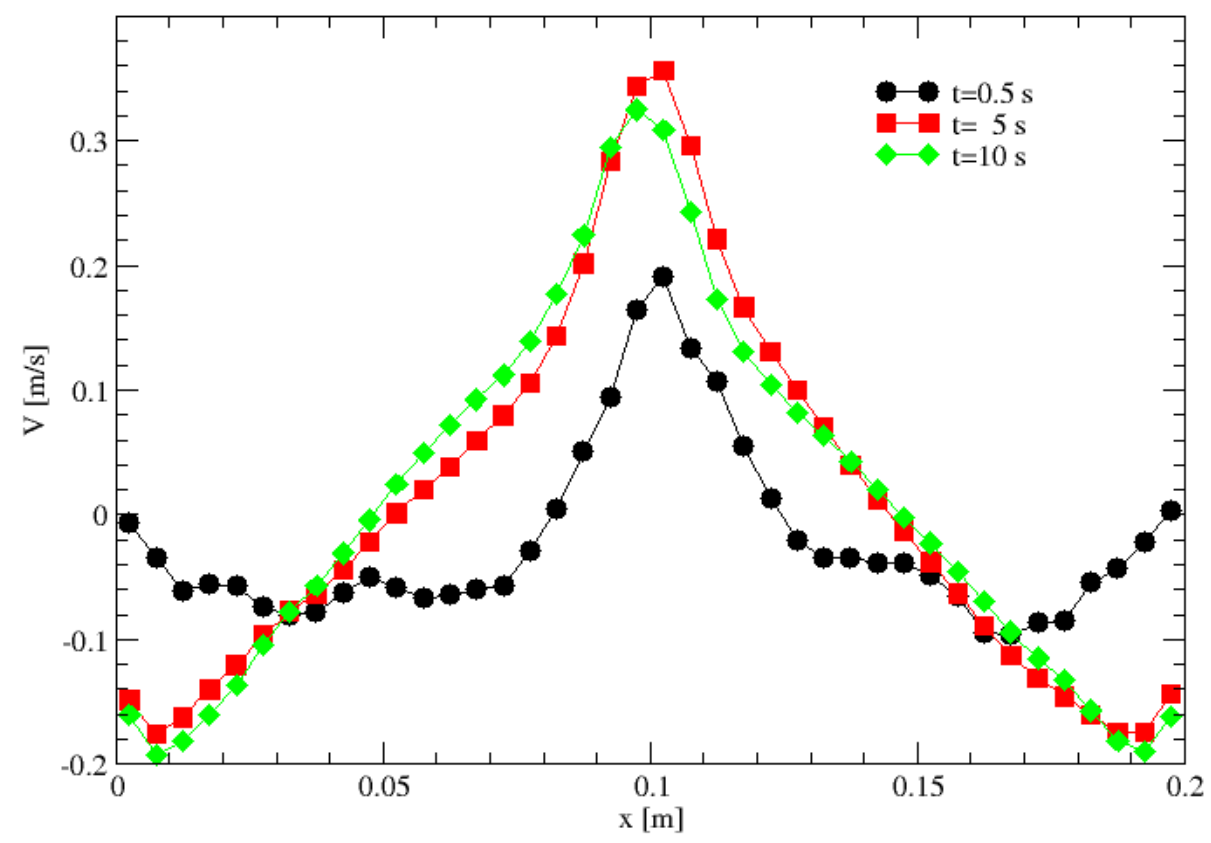

Figure 2: Particles slip velocity at the wall in a jet flow

We have improved the efficiency of our DNS code and demonstrated that it can handle a large number of particles. Figure 3 shows the snapshots of the fluidizations of 10,000 particles at different time. The rise of fluidization "bubbles" is evident in the figure.

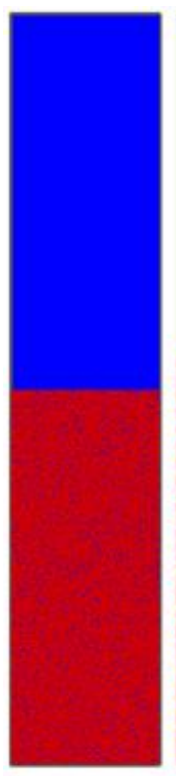

$\mathrm{t}=0 \mathrm{~s}$

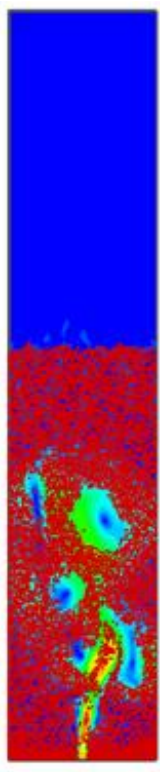

$\mathrm{t}=1 \mathrm{~s}$

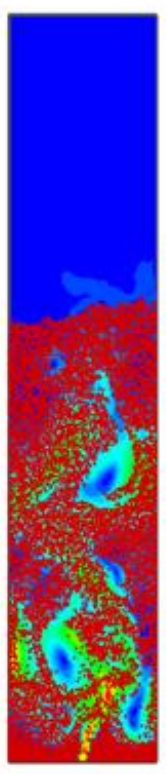

$\mathrm{t}=2 \mathrm{~s}$

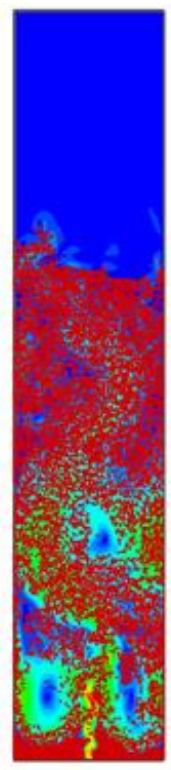

$\mathrm{t}=3 \mathrm{~s}$

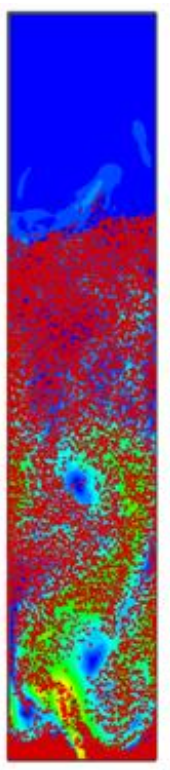

$\mathrm{t}=4 \mathrm{~s}$

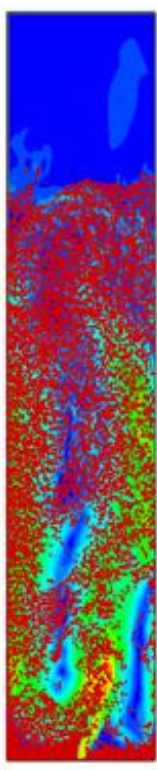

$\mathrm{t}=5 \mathrm{~s}$

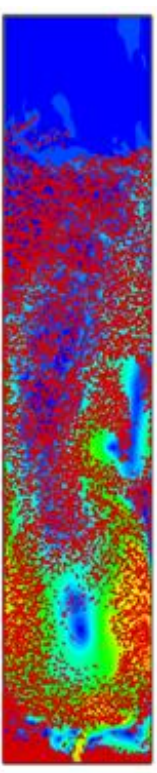

$\mathrm{t}=6 \mathrm{~s}$

Figure 3: Snapshots of particles fluidization by a jet flow 
Our three-dimensional DNS code has been used to study the particle-wall collision in a viscous fluid. The bouncing motion of spherical particles falling onto a solid plate under gravity in a viscous fluid has been investigated experimentally by Gondret et al. (2002). They used particles of different sizes and different materials including carbide tungsten, stainless steel, soda glass, and nylon. The fluids used in their experiment are air, water, and silicone oil of different viscosity. They recorded the trajectory and velocity of a particle during setting and bouncing motion in a fluid. Figure 4(a) shows the experimentally measured trajectory of a sphere (diameter is 3mm) impacting a solid wall in silicon oil RV10. ${ }^{1}$ By choosing proper model parameters for soft-sphere collision scheme, we are able to reproduce their results numerically, as shown in Figure 4(b).

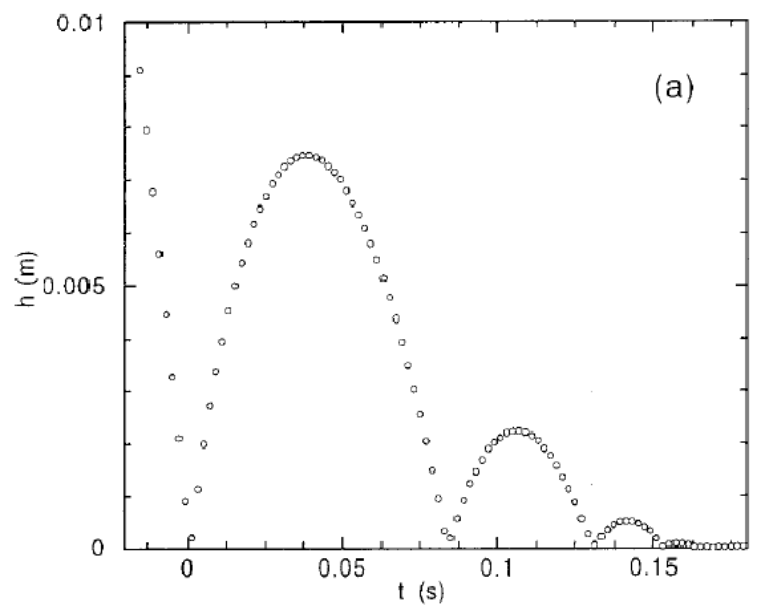

(a)

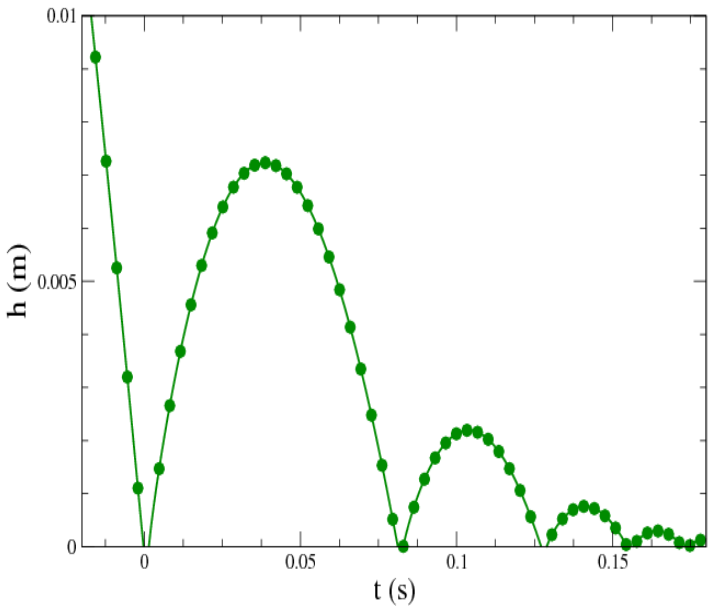

(b)

Figure 4: Particle trajectory as a function of time for a $3 \mathrm{~mm}$ steel sphere in silicon oil RV10. (a) Experimental measurement by Gondret et al. ${ }^{1}$; (b) Present numerical simulation ( $k=317,000 \mathrm{dyn} / \mathrm{cm}, \eta=14$ dyn.s $/ \mathrm{cm}$.)

One of the research goals is to identify the relationships between model parameters of collision (spring stiffness, damping coefficient, and friction coefficient) and the physical parameters of collision (Reynolds number of flows, properties of fluid and particle, etc al.). We can achieve this goal by assessing the results of velocity and trajectory of a particle in collision obtained from experimental measurements and numerical simulations.

One of the research goals of the proposal is to apply the full resolved DNS approach to study particle-particle interactions and derive closure laws that can be employed in other scale models such as the TFM of MFIX.

It is well known that the effect to the dynamics of a particle is strongly influenced by the presence of neighboring particles. In this quarter, we investigated the settling of a heavy particle in the presence of a system of surrounding neutral particles. The simulations we conducted here use a fluid of viscosity $0.058 \mathrm{~kg} / \mathrm{m} . \mathrm{s}$, and density $960 \mathrm{~kg} / \mathrm{m} 3$; the particle has a diameter of 
$15 \mathrm{~mm}$, and its density is $1500 \mathrm{~kg} / \mathrm{m} 3$. The simulation domain is a $9 \mathrm{cmx} 9 \mathrm{cmx} 45 \mathrm{~cm}$ box with periodic boundary conditions applied on all sides. By randomly distributing different numbers of neutral particles of the same size (shown in Figure 5), we are able to investigate the dynamics of the heavy particle in a suspension of different solid fraction.

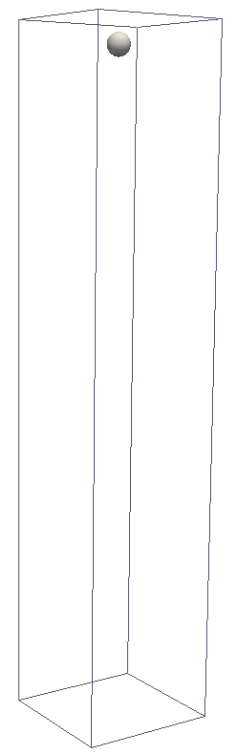

(a)

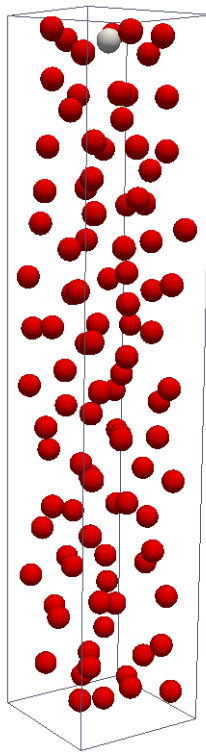

(b)

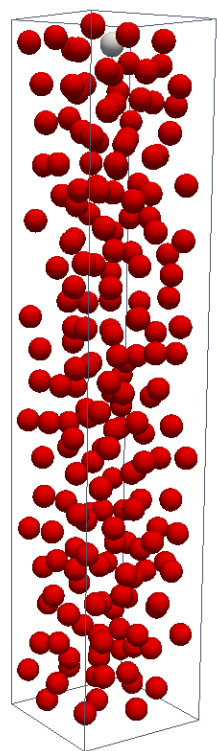

(c)

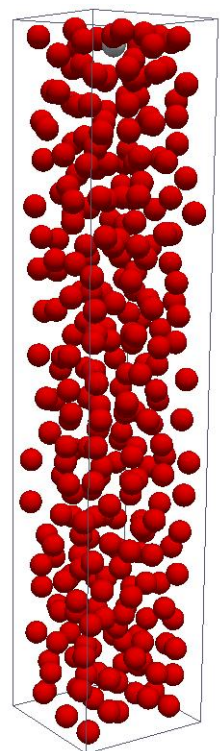

(d)

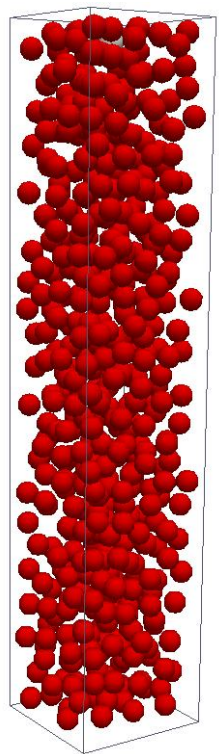

(e)

Figure 5: The settling of a heavy particle (in white color) in the presence of neutral particles (in red color) suspended in a fluid at different solid fractions: (a) 0 ; (b) $5 \%$; (c) $10 \%$; (d) $15 \%$; (e) $25 \%$.

The presence of neutral particles in flows increases the resistance and makes the values of the drag force and the collision force higher; as a result, it decreases the velocity of the settling particle. Figure 6(a) shows the setting velocity of the heavy particle at different solid fractions. It is seen that the settling velocity in the presence of neighboring particles fluctuates with respect to time mainly due to its interactions with surrounding particles. The mean velocity of the heavy particle, however, can be extracted from the slope of its vertical trajectory component vs. time graph, as shown in Figure 6(b). 


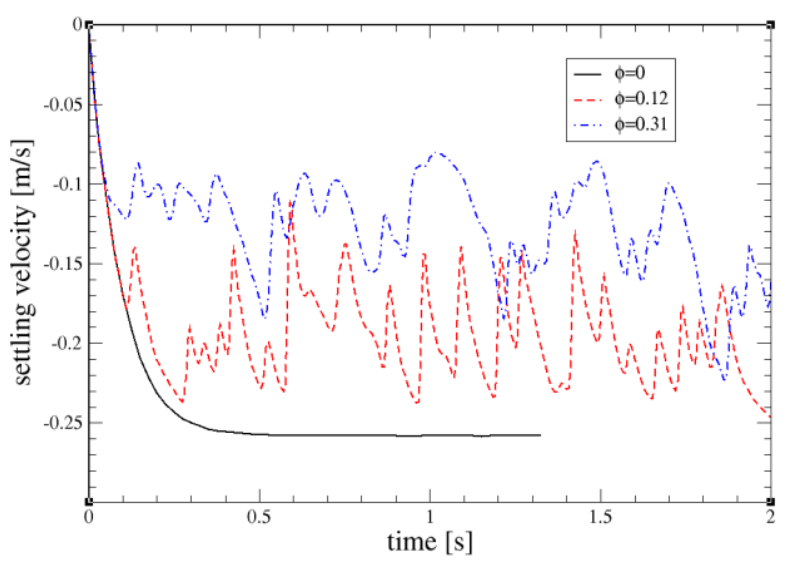

(a)

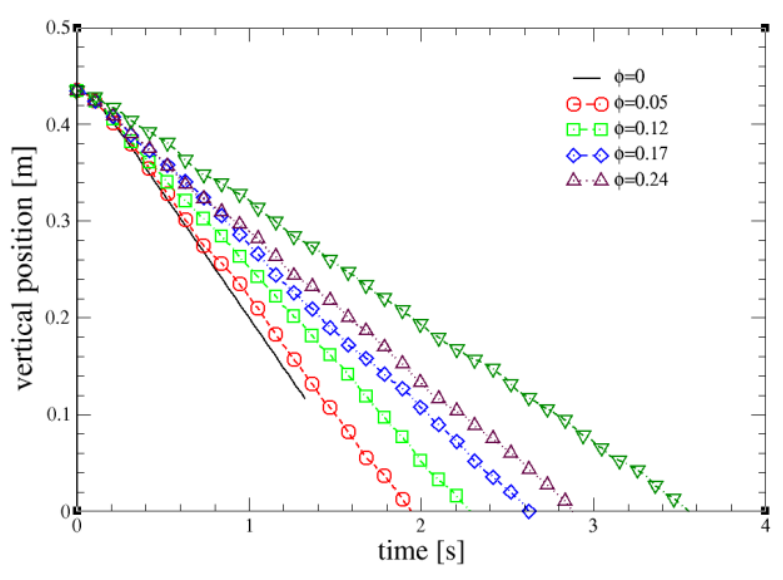

(b)

Figure 6: Setting of a heavy particle in the presence of surrounding particles. (a) vertical velocity vs. time (b) vertical position vs. time.

Table 1 shows the terminal velocity of the heavy particle in flows with a variety of solid fraction. These data are plotted in Figure 8 and a good correlation is found to be:

$\frac{U_{t, \phi}}{U_{t, 0}}=1-1.41 \phi^{0.85}$

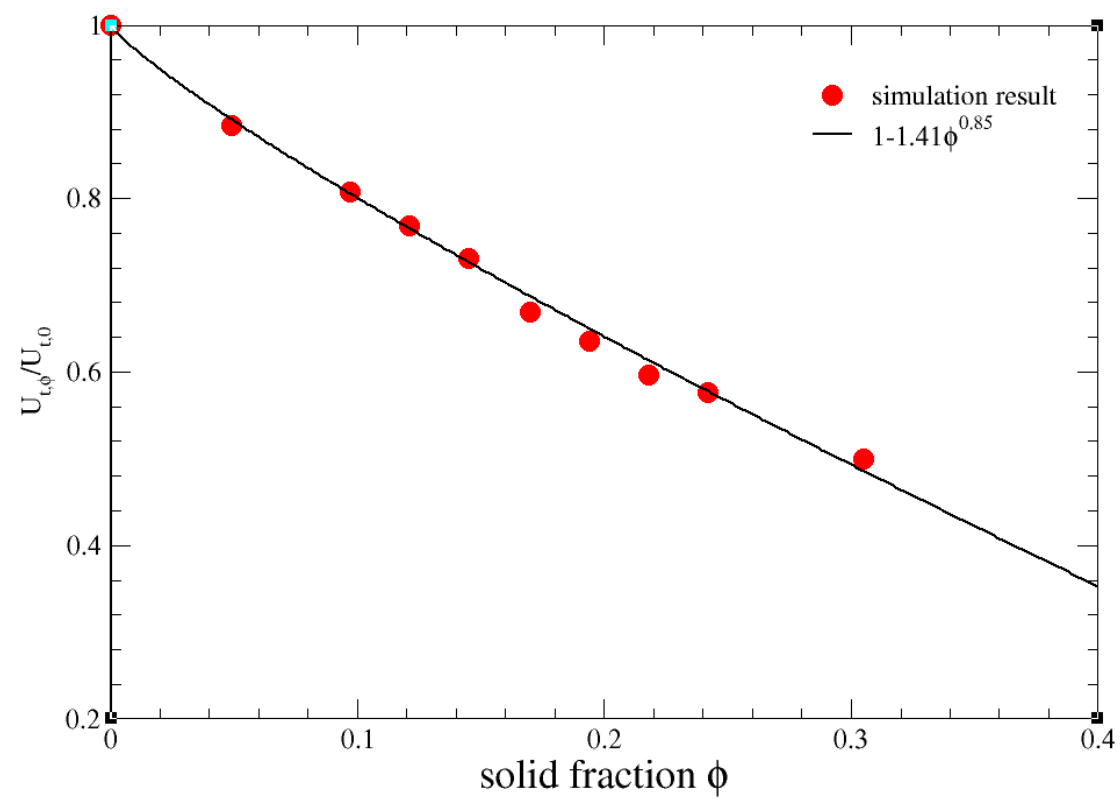

Figure 7: Particle's settling velocity in flows of different solid fraction.

Table 1: particle terminal velocity vs. solid fraction 


\begin{tabular}{cccc}
$\begin{array}{c}\text { Number of surrounding } \\
\text { particles }\end{array}$ & Solid fraction & \multicolumn{2}{c}{ Terminal velocity $(\mathrm{m} / \mathrm{s})$} \\
0 & & Simulated & Correlated \\
100 & 0.049 & 0.260 & 0.260 \\
200 & 0.097 & 0.230 & 0.232 \\
250 & 0.121 & 0.210 & 0.210 \\
300 & 0.145 & 0.200 & 0.200 \\
350 & 0.170 & 0.190 & 0.189 \\
400 & 0.194 & 0.174 & 0.179 \\
450 & 0.218 & 0.165 & 0.169 \\
500 & 0.242 & 0.155 & 0.160 \\
630 & 0.305 & 0.150 & 0.150
\end{tabular}

The effects of the thermal interaction between a particle and a fluid have two aspects: the motion of fluid affects the heat transfer and energy balance of a particle; and the heat transfer from particles influences the fluid motion via the buoyancy effect. One of the important parameters in the energy equation used in the discrete element method is the Nusselt number, which depends on the flow velocity field and the fluid properties. Almost all the results in literature are based on the heat transfer of a single solid particle in an infinite domain with uniform cross flow velocity. In order to have a more accurate energy equation that can be applied for modeling the heat transfer of particulate flows, numerical studies are needed to account for the influence of effects such as non-uniform velocity field, particle shape, solid fraction, and wall effects. We have developed a DNS code with an added thermal-interaction module that can account these effects. When the temperature of particle and its surrounding fluid is not the same, heat is transferred between the particles and the fluid. The heat flux influences the properties of the surrounding fluid and changes the dynamics of the sedimentation of the particles. By using the DNS, we have examined the effects of the thermal interaction on the sedimentation velocity and the drag coefficient of a single particle settling in a fluid. 


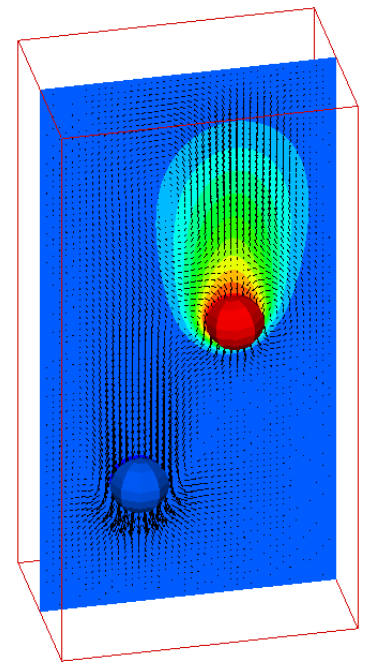

(a) Snapshot at $t=0.85$ s for $G r=1000$

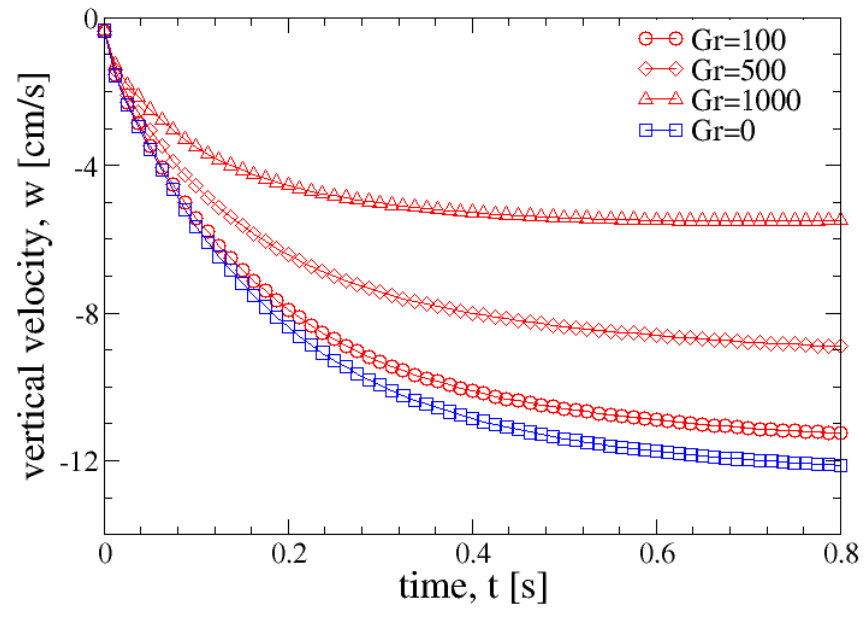

(b) vertical velocity at different $\mathrm{Gr}$ numbers

Figure 8: Free fall of spheres in a liquid. Liquid: density $\rho_{\mathrm{f}}=0.96 \mathrm{~g} / \mathrm{cm}^{3}$, viscosity $\eta_{\mathrm{f}}=0.58 \mathrm{P}$, Prandtl number $\operatorname{Pr}=0.7$; Particle: density $\rho_{\mathrm{p}}=1.17 \mathrm{~g} / \mathrm{cm}^{3}$, radius a $=0.75 \mathrm{~cm}$.

Figure 8 (a) shows a snapshot at $\mathrm{t}=0.86 \mathrm{~s}$ of two spheres falling in a liquid column. The two spheres are initially positioned at the same level. Figure 8(b) shows the settling velocity of a sphere at four different Grashof numbers. It is seen again that the velocity of the hot particle with $\mathrm{Gr}=1000$ is less than half compared to the case of an isothermal particle $(\mathrm{Gr}=0)$.

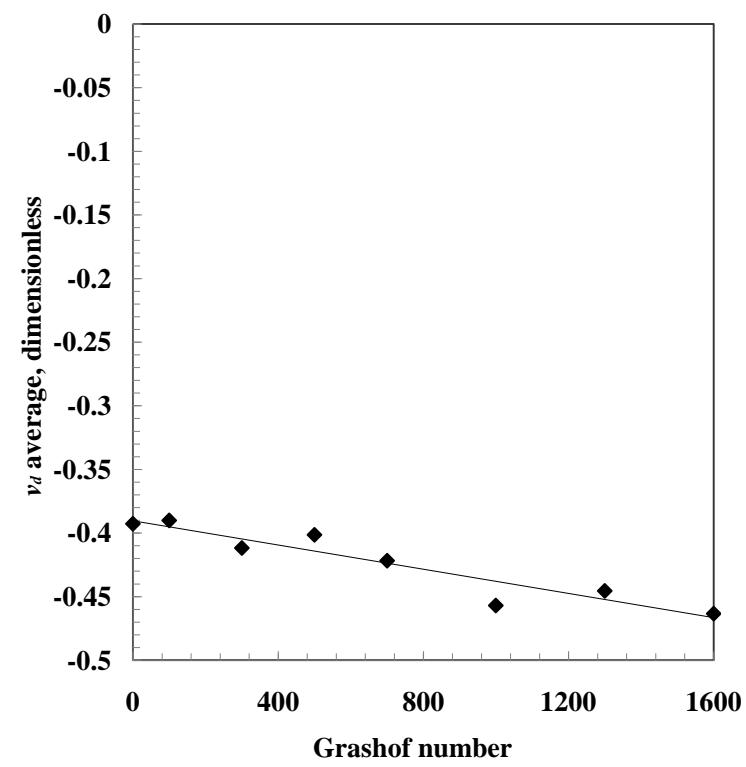

Figure 9: Influence of Grashoff number to the dimensionless setting velocity at the wall.

The effect of temperature gradient to dynamics of particles near a solid wall has also been 
investigated. From the many particles in our simulations, we isolated the particles that are close to the vertical walls and performed an analysis of their vertical velocities. From the extrapolation of the resulting data, we compute the value of the vertical velocity of the particles at the point where the particle boundary condition at the wall is defined. This is at a distance of one particle radius from the wall $(\mathrm{y}=\mathrm{d} / 2)$. we concluded that the best way to define the boundary condition is using a dimensionless vertical velocity for the particles, which is as follows:

$v_{d}=\frac{v_{p}-V_{f}}{V_{T}}$,

where $v_{p}$ is the particle vertical velocity obtained from the simulations, $\mathrm{v}_{\mathrm{T}}$ is the terminal velocity of the particle and $v_{f}$ is the globally averaged velocity of the fluid:

$V_{f}=V_{\text {jet }} \frac{A_{\text {jet }}}{A_{\text {bottom }}}$

The influence of the Grashoff number on these suspended in the flow field is shown in Figure 9, where it is observed that the heat transfer does influence the interaction velocity of the particles.

\section{CONCLUSIONS}

We have developed a DNS method that is capable to simulate the motion of a large number of particles in a viscous fluid. We used the soft-sphere scheme for both particleparticle and particle-wall collision. From the simulation results of the fluidization process of 500 circular particles in a two-dimensional fluidized bed reactor using five different fluidization velocities ranging from $0.10 \mathrm{~m} / \mathrm{s}$ to $0.20 \mathrm{~m} / \mathrm{s}$, we found that the fluidization velocity and fluid fraction is nicely correlated by a power-law with exponent coefficient $\mathrm{n}=4.24$; the extended fluidization velocity at zero solid fraction (blowout velocity) is close to the sedimentation velocity of a single particle. We observed that there is significant downward slip velocity of particles at a vertical wall. The no-slip boundary condition is not applicable for the solid phase when the two-fluid model is used. A slip boundary condition should be employed instead, based on a slip length, which may be calculated from our simulations. Using spatial and time-averaging techniques, we are able to obtain the velocity profiles for solid particles and extract slip lengths and slip coefficients at a wall. Our simulation results show that the slip velocity increases as the fluidization velocity increases. However, the slip length and the slip coefficient appear to be insensitive to the fluidization velocity and remain nearly constant with approximate values of $-1.2 d$ and $-167 / \mathrm{m}$. Thus, a slip boundary condition may be applied for the solid phase in two-phase models that would not depend on the flow rate of fluid. 
We have also studied the behaviour of small particles with diameters in the range 4 to $12 \mathrm{~mm}$ close to vertical walls. It was observed that the particles tend to aggregate close to the impermeable vertical walls. Inter-particle collisions in the vicinity of the walls become very important in the particulate motion and affect significantly the trajectories of individual particles and their vertical velocities. Secondly, the time- and ensemble-average relative vertical velocity of the particles at the wall region was calculated by an interpolation of the time-averaged numerical data. It was observed that the average relative dimensionless vertical velocity for all the particles or for the fully suspended particles depends strongly on the size of the particles and actually increases with the size of the particles. All the numerical data and their averages point to the fact that, at the wall, which

for the particles is defined at a distance $d / 2$ from the actual wall, the particles maintain a fraction of their terminal velocity. There is partial slip at the wall in the tangential direction, which appears to be in the range -0.45 to -0.29 .

\section{REFERENCES}

Alder, B. J. and Wainwright, T. E., 1957. Phase Transition for a Hard-Sphere System, J. Chem. Phys. 27:1208.

Alder, B. J. and Wainwright, T. E., 1959. Studies in Molecular Dynamics. I. General Method, J. Chem. Phys. 31:459.

Balevičius, R., Kačianauskas, R., Mróz, Z., and Sielamowicz, I., 2008. Discrete-particle investigation of friction effect in filling and unsteady/steady discharge in three-dimensional wedge-shaped hopper, Powder Technology 187:159-174.

Crowe, C. T., 1982. Review-numerical methods for dilute gas particle flows, ASEME J. Fluids Eng. 104: 297-303.

Cundall, P.A. and Strack, O. D. L., 1979. A discrete numerical model for granular assemblies, Géotechnique 29:47.

Drew, D. and Lahey, R. Jr., 2001. The analysis of two-phase flow and heat transfer using a multidimensional, four field, two-fluid model, Nuclear Eng. and Design 204: 29-44.

Feng, J. , Hu, H. H. and Joseph, D. D., 1994. Direct simulation of initial value problems for the motion of solid bodies in a Newtonian fluid Part 1. Sedimentation, J. Fluid Mech. 261:95-134.

Feng, Z.-G. and Michaelides, E. E., 2004. The immersed boundary-lattice Boltzmann method for solving fluid-particles interaction problems, J. Comput. Phys. 194: 602-628.

Final Report for Grant No. DE-NT0008064 
Feng, Z.G. and Michaelides, E. E., 2005. Proteus: a direct forcing method in the simulations of particulate flows, J. Comput. Phys. 202:20-51.

Feng, Z.G. and Michaelides, E.E., 2008. Inclusion of heat transfer computations for particle laden flows, Phys. Fluids 20:1-10.

Feng, Z.G. and Michaelides, E.E., 2009a. Heat transfer in particulate flows with Direct Numerical Simulation (DNS), Int. J. Heat and Mass Transfer 52:777-786.

Feng, Z.G. and Michaelides, E.E., 2009b. Robust treatment of no-slip boundary condition and velocity updating for the lattice-Boltzmann simulation of particulate flows, Computers \& Fluids 38: $370-381$.

Feng, Z.G., Michaelides, E. E., and Mao, S.L., 2010. A Three-Dimensional Resolved Discrete Particle Method for Studying Particle-Wall Collision in a Viscous Fluid, ASME J. of Fluids Eng. 132: $967-973$.

Gondret, P, Lance, M., and Petit, L., 2002. Bouncing motion of spherical particles in fluids, Phys. Fluids, 14:643-652.

Glowinski, R., Pan, T.-W. , Hesla, T. I., and Joseph, D. D., 1999. A distributed Lagrange multiplier/fictitious domain method for particulate flows. Int. J. Multiphase Flow 25: 755-794.

Hu, H. H., Joseph, D. D. , and Crochet, M. J., 1992. Direct numerical simulation of fluid particle motions, Theoret. Comput. Fluid Dynamics 3: 285-306.

Ishii, M., 1975. Thermo-fluid dynamic theory of two-phase flow, Eyrolles.

Johnson, P. C. and Jackson, R., 1987. Frictional-collisional constitutive relations for granular materials, with application to plane shearing J. Fluid Mech., 176:67-93.

Kalthoff, W., Schwarzer, S., and Hermann, H. J., 1996. Algorithm for the simulation of particle suspensions with inertia effects Physics Review E., 56: 2234-2242 (1996).

Kuo, H. P., Knight, P. C., Parker, D. J., Tsuji, Y., Adams, M. J., and Sevill, J. P. K., 2002. The influence of DEM simulation parameters on the particle behaviour in a V-mixer, Chemical Engineering Science 57:3621-3638.

Ladd, A. J. C., 1994a. Numerical simulations of particulate suspensions via a discretized Boltzmann equation. Part I. Theoretical foundation J. Fluid Mech., 271: 285-310

Ladd, A. J. C., 1994b. Numerical simulations of particulate suspensions via a discretized Boltzmann equation. Part II: Numerical results, J. Fluid Mech. 271: 311-339 (1994). 
Lauga, E. Brenner, M. P. and Stone, H.A., 2007. Microfluidics: The no-slip Boundary Condition, ch. 9, in Experimental Fluid Mechanics, eds. Tropea, C., Yarin, A. and Foss, J.F., Springer, Berlin.

Michaelides, E. E., 2006. Particles, bubbles and drops: their motion, heat and mass transfer, World Scientific Publishing.

Pan, T.-W. Joseph, D.D., Bai, R., Glowinski, R. , and Sarin, V., 2002. Fluidization of 1204 spheres:simulation and experiment, J. Fluid Mech. 459:161-191.

Peskin, C. S., 1977. Numerical analysis of blood flow in the heart, J. Comput. Phys. 25: 220252.

Renzo, A. Di and Maio, F. P. Di., 2004. Comparison of contact-force models for the simulation of collisions in DEM-based granular flow codes, Chemical Engineering Science 59:525-541.

Schäfer, J. , Dippel S. , and Wolf, D. E., 1996. Force schemes in simulations of granular materials, J. Phys. I France 6:5.

Sommerfeld, M. and Huber, N., 1999. Experimental Analysis and Modeling of Particle-Wall Collisions, Int. J. Multiphase Flow 25:1457-1489.

Tsuji, Y., Kawaguchi, T., and Tanaka, T., 1993. Discrete particles simulation of 2-dimensional fluidized bed, Powder Technology 77:79-87.

Uhlmann M., 2005. An immersed boundary method with direct forcing for the simulation of particulate flows, J. Comput. Phys. 209:48-476.

van der Hoef, M.A., van Sint Annaland, M., Deen, N. G , and Kuipers, J. A. M., 2008. Numerical simulation of dense gas-solid fluidized beds: a multiscale modeling strategy, Annu. Rev. Fluid Mech. 40:47-70.

Yu, Z., Shao, X., and Wachs, A., 2006. A fictitious domain method for particulate flows with heat transfer, J. Comput. Phys. 217: 424-452.

Zhang, Z. and Prosperetti, A., 2005. A second-order method for three-dimensional particle simulation, J. Comput. Phys. 20:292-324.

\section{LIST OF PUBLICATIONS}

\subsection{Archival Publications:}

Kartushinski, A., Michaelides, E. E., Rudi, Y., and Graham, N., 2010, "RANS Modeling of a particulate turbulent round jet," J. of Chemical Engineering Science, vol. 65, pp. 3384-3393.

Final Report for Grant No. DE-NT0008064 
Feng, Z.G., Michaelides, E. E. and Mao, S.L., 2010, “A Three-Dimensional Resolved Discrete Particle Method for Studying Particle-Wall Collision in a Viscous Fluid," J. of Fluids Eng., vol. 132, number 091302, 2010.

Michaelides, E. E., and Roig, A., 2010, "A Reinterpretation of the Odar and Hamilton Data on the Unsteady Equation of Motion of Particles," A.I. Ch. E. Journal, DOI 10.1002/aic.12498, 2010.

Kartushinski, A., Michaelides, E. E., Rudi, U. A., Shcheglov, I. N. , and Tisler, S. V., 2011, "Numerical Simulation of Three-Dimensional Gas-Solid Particle Flow in a Horizontal Pipe," A. I. Ch. E. Journal, DOI 10.1002/aic.12528.

A.P. Davis, E. E. Michaelides, and Z.-G. Feng, 2011, Particle velocity near vertical boundaries - A source of uncertainty in two-fluid models, Powder Technol., doi:10.1016/j.powtec.2011.09.031

Yang, B. J., Mao, S. L., Altin, O., Feng, Z. G. and Michaelides, E. E., 2011, "Condensation Analysis of Exhaust Gas Recirculation (EGR) Cooler for Heavy-Duty Trucks," Applied Thermal Engineering, volume 3, doi:10.1115/1.4004745.

Feng, Z.-G., Michaelides, E. E. and Mao, S.-L., 2012, "On the drag force of a viscous sphere with interfacial slip at small but finite Reynolds numbers," Fluid Dynamics Research, Fluid Dyn. Res. 44025502 doi:10.1088/0169-5983/44/2/025502.

Feng, Z.-G. and Michaelides, E. E., 2012, "Heat transfer from a nano-sphere with temperature and velocity discontinuities at the interface," Int. J. Heat and Mass Transf. 55:6491-6498.

Michaelides, E. E., 2012, "Entropy Production and Optimization of Geothermal Power Plants," J. of Non-Equilibrium Thermodynamics, 37, pp. 233-246.

Feng, Z.-G., Michaelides, E. E. and Mao, S.-L., 2012, "Using the Direct Numerical Simulation to Compute the Slip Boundary Condition of the Solid Phase in Two-Fluid Model Simulations," Powder Technology (submitted).

Musong, S.G. and Feng Z.-G., 2012, "Effect of Model Parameters of Soft-Sphere Collision Scheme to the Particle-Particle Collision in a Viscous Fluid," Powder Technology (submitted).

\subsection{Conference Publications and Presentations:}

A. P. Davis, Z-G Feng and E. E. Michaelides, "Application of the Immersed Boundary Method and Direct Numerical Simulation for the Heat Transfer from Particles," Proceedings of the 2009 ASME-FEDSM, meeting of the Division of Fluids Engineering at Veil, CO.

Adelina P.Davis, Efstathios E. Michaelides and Zhi-Gang Feng, "Particle Velocity near Vertical Boundaries - A source of Uncertainty in Two-Fluid Models" 7th International Conference on 
Multiphase Flow, ICMF 2010, Tampa, FL, May 30 - June 4, 2010.

Roig, A. and Michaelides, E.E., "A re-interpretation of the Odar and Hamilton data on the history terms of the equation of motion,” ICMF-2010, May 2010, Tampa, FL.

(keynote presentation) Michaelides, E. E. and Feng, Z.-G., "Direct Numerical Simulations of Particulate Flows that Include Momentum, Heat and Mass Exchanges" ICMF-2010, May 2010, Tampa, FL.

Alexander Kartushinsky and Efstathios E. Michaelides, "RANS Modeling of a Particulate Turbulent Downward Jet," 7th International Conference on Multiphase Flow, ICMF 2010, Tampa, FL, May 30 - June 4, 2010.

Feng, Z.-G. and Michaelides, E. E., 2010, "Simulation Of The Particle-Wall Collisions In A Viscous Fluid Using A Resolved Discrete Particle Method," Proceedings of the ASME 3rd Joint US-European Fluids Engineering Summer Meeting, FEDSM-ICNMM 2010, August 1-5, 2010, Montreal, Canada.

Feng, Z-G, Michaelides, E. E., and Mao, Shaolin, 2011,“A multilevel simulation approach to derive the slip boundary condition of the solid phase in two-fluid models," 64th Annual Meeting of the APS Division of Fluid Dynamics, Baltimore, Maryland. November, 2011.

Feng, Z-G, and Samuel, G.M., "The Effect of Neighboring Particles on the Dynamics of a Particle in a Viscous Fluid," NETL 2012 Conference on Multiphase Flow Science, NRCCE, Morgantown, WV. May 22-24.

Feng Z-G, Musong, S.G. and Michaelides, E.E., "Effect of Model Parameters of Soft-Sphere Collision Scheme to the Particle-Particle Collision in a Viscous Fluid," NETL 2012 Conference on Multiphase Flow Science, NRCCE, Morgantown, WV. May 22-24.

Feng, Z-G, and Samuel, G.M., "The Effect of Neighboring Particles on the Dynamics of a Particle in a Viscous Fluid," NETL 2012 Conference on Multiphase Flow Science, NRCCE, Morgantown, WV. May 22-24.

Feng, Z-G, Feng, Y, and M. Anderssion, "Shape effects on the drag force and motion of particles in a viscous fluid at low Reynolds numbers," ASME IMECE 2012-89469, Houston, 2012.

Feng, Z-G, Solan, G., Bhaganagar, K., and Banerjee, D., "Numerical simulation of nanoparticle clustering with experimental validations," the $65^{\text {th }}$ Annual Meeting of the APS Division of Fluid Dynmaics, November, San Diego, 2012.

Feng, Z-G and Andersson, M., "Modeling flows in porous media using immersed boundary based lattice Boltzmann method," ASME IMECE 2012-89427, Houston, 2012.

Michaelides E.E. and Feng, Z-G., 2012, "A DNS method for particle motion to establish 
boundary conditions in coal gasifiers,” ECOS-2012, Perugia, Italy, June 2012.

\section{PERSONALS PARTICIPATED IN THE PROJECT}

The following are the personals who have been participated in the project and also finically supported by the project.

PI and co-PI:

Zhi-Gang Feng, Assistant Professor, Department of Mechanical Engineering., University of Texas at San Antonio (UTSA);

Efstathios E. Michaelides, Professor, Department of Mechanical Engineering, Texas Christian University.

Graduate students (UTSA):

Adelina Davis,

Samuel A. Yochmowitz,

Gem Musong Samuel,

Maria Andersson.

Undergraduate student (UTSA):

Steven Cook,

Sergio Gonzalez,

Eric Stewart 